\title{
Premiers éléments de définition d'un type émergent de FASP : l'ecothriller
}

\section{Camille Biros}

\section{(2) OpenEdition}

1 Journals

Édition électronique

URL : http://journals.openedition.org/asp/963

DOI : $10.4000 /$ asp.963

ISBN : 978-2-8218-0412-8

ISSN : 2108-6354

\section{Éditeur}

Groupe d'étude et de recherche en anglais de spécialité

Édition imprimée

Date de publication : 30 mars 2010

Pagination : 67-85

ISSN : 1246-8185

\section{Référence électronique}

Camille Biros, « Premiers éléments de définition d'un type émergent de FASP : l'ecothriller », ASp [En ligne], 57 | 2010, mis en ligne le 01 mars 2013, consulté le 03 novembre 2020. URL : http:// journals.openedition.org/asp/963; DOI : https://doi.org/10.4000/asp.963

Ce document a été généré automatiquement le 3 novembre 2020

Tous droits réservés 


\title{
Premiers éléments de définition d'un type émergent de FASP : l'ecothriller
}

\author{
Camille Biros
}

\section{Introduction}

Dans l'article fondateur des études sur la fiction à substrat professionnel (FASP) dans le cadre de la recherche en anglais de spécialité, Michel Petit évoque à titre d'exemple le "legal thriller », le " medical thriller », le « military thriller », le " adventure thriller » et le « technical thriller " (Petit $1999: 57)$. L'« ecothriller »ne figure donc pas dans la liste de ce qui peut être considéré comme FASP. Cette absence pourrait s'expliquer tout simplement par la date à laquelle cet article a été écrit, 1999, date à laquelle l'ecothriller demeurait encore un genre confidentiel, bien que plusieurs fictions de ce type eussent déjà été écrites. Certains situent l'émergence de l'ecothriller en tant que genre vers la fin des années 1990. Dans un article de juin 2007 publié dans Library Journal, on peut lire : « One of the newest fiction subgenres emerging over the last decade is ecothrillers " (Schollmeyer 2008). Pourtant le succès de ce genre encore modeste n'est que récent - sans doute a-til été porté par les préoccupations écologiques grandissantes de l'opinion publique relayée par les médias.

2 Pour la présente tentative de définition du genre, nous avons retenu un corpus composé d'une vingtaine de romans. Un passage en revue de leur résumé, de leur couverture cartonnée et de critiques à leur sujet, nous a permis de les sélectionner comme étant représentatifs du genre. Nous nous sommes concentrée sur l'étude des paratextes de ces romans et de la littérature critique les concernant, et nous avons procédé à la lecture et à l'analyse d'un certain nombre d'entre eux. La liste non exhaustive d'ecothrillers qui composent notre corpus peut être consultée dans la section bibliographique. Une lecture rapide de cette liste permet d'observer que, sur vingt-etun romans, seuls trois ont été publiés avant l'année 2000 et seulement huit avant 2005. 
Le succès récent et indéniable du genre peut également être mis en évidence par la conversion à l'écriture de romans de ce genre par des auteurs qui étaient jusqu'alors connus pour écrire d'autres types de thriller. Michael Crichton, considéré avant tout comme le fondateur du technothriller ${ }^{1}$, en offre un bon exemple puisqu'il a produit un roman que l'on peut qualifier d'« ecothriller» en 2005 avec sa fiction State of Fear.

La nouveauté du succès du genre peut s'expliquer par une référence à l'actualité. Il semble en effet que des phénomènes de prise de conscience de l'importance des questions écologiques ont eu lieu récemment. On peut par exemple évoquer l'impressionnante médiatisation de différentes catastrophes écologiques (tsunami en Asie du Sud-Est, canicule en Europe du Nord, ouragan Katrina) ainsi que la diffusion de documents suggérant l'ampleur des conséquences possibles du réchauffement climatique comme la publication très commentée du rapport Stern (Stern 2006) et le film au succès étonnant présentant le point de vue d'Al Gore sur le réchauffement climatique (Guggenheim 2006). Ces phénomènes semblent avoir conduit le public à un intérêt renouvelé pour tout ce qui a trait aux problématiques environnementales, intérêt qui a été aisément exploité par les auteurs de fiction. Le rapport particulier au réel que M. Petit analyse dans son article sur la FASP, qui montre qu'un roman de FASP « accorde [...] une large place à la représentation des divers éléments qui composent ce réel, personnes, objets, procédures » (Petit 1999 : 68), explique que la FASP est peut-être plus influencée par ce qui se passe dans le monde réel que les autres types de fiction. Des romans de science-fiction ayant pour sujet la fin du monde (catastrophe thrillers), ont été écrits depuis bien longtemps, mais avec la crise écologique qui prend actuellement une importance considérable dans la société, les auteurs de FASP n'ont plus besoin de tout inventer. Il s'agit bien de s'appuyer sur des éléments scientifiques et technologiques réels pour construire le contexte des thrillers. L'auteur de cet article s'interroge tout d'abord sur les raisons pour lesquelles le genre de l'ecothriller peut être considéré comme un nouveau type de FASP. Elle tente ensuite de définir ses caractéristiques principales avant de se poser la question des rapports entre le réel fictionnel des FASP et le réel prédit par la science dans le contexte actuel. Les ecothrillers pourraient avoir un effet sur le monde réel car ils permettent la représentation d'une catastrophe écologique qui reste, sans ce type de représentation, du domaine de l'impensable.

\section{L'ecothriller, un nouveau type de FASP?}

Lorsqu'il définit les caractéristiques récurrentes des différentes fictions à substrat professionnel, M. Petit établit une distinction entre les caractéristiques externes, liées aux conditions dans lesquelles les romans sont publiés, et les caractéristiques internes qui permettent de définir le genre. Nous allons commencer ici par considérer les caractéristiques externes. Parmi celles-ci, il faut évoquer le fait que les ouvrages de FASP aspirent à devenir des bestsellers (Petit 1999: 58). Les différents ouvrages considérés ici répondent bien à cette description. Des occurrences du mot "bestseller », ou de mots appartenant à la même famille, se retrouvent ainsi dans le paratexte ${ }^{2}$ d'un nombre élevé de nos ouvrages. En première page de couverture de The Swarm (Schatzing 2006), figure : " the two million copy international bestselling phenomenon » et sur celle de The Last Albatros (Irvine 2000) : « a compelling thriller from internationally bestselling author ». Les exemples possibles sont multiples. Les ecothrillers semblent donc 
bien respecter ce premier critère de la FASP. Ils visent à conquérir un large public plutôt qu'un public de spécialistes. Le fait que la diffusion de ce genre soit visiblement plus importante à la suite de l'intérêt croissant pour la crise écologique chez le grand public, peut sembler signifiant de ce point de vue.

\subsection{L'ecothriller, genre émergent}

Lorsqu'il définit en quoi la FASP peut être qualifiée de "genre à succès international » (1999: 60), M. Petit met en évidence le fait que certains éléments du paratexte permettent de mettre en relation l'ouvrage avec d'autres ouvrages du même genre, montrant par là que le genre est cristallisé et aisément identifiable par le public. Ce n'est pas le cas des ouvrages étudiés ici. Il semble que le genre de l'ecothriller est apparu trop récemment pour que ce critère de définition de la FASP s'applique. La seule référence à un autre auteur se trouve sur la couverture de Freezing Point de Karen Dionne. La critique suivante y figure: "Karen Dionne is the new Michael Crichton». M. Crichton est surtout connu comme le père du technothriller et c'est probablement une référence à ce genre que l'on trouve ici.

6 Il est possible que la raison pour laquelle la plupart des romans étudiés ici ne comprennent pas le terme ecothriller dans le paratexte est que le genre n'est pas encore suffisamment connu pour avoir été assimilé par un large public. Il existe quelques exceptions notables. Ainsi Zodiac (Stephenson 1988) a pour sous-titre «the ecothriller ", soulignant clairement son appartenance au genre. Pour ce qui est de Dark Rain (Corderoy 2006), on peut également dire qu'il est visiblement présenté comme un ecothriller puisqu'on trouve dans ses critiques en début d'ouvrage la phrase suivante : « An energetic and agreeable example of the ecothriller» (Corderoy $2006: 4)$. Dans d'autres cas, ce qualificatif est absent.

7 Le terme pourrait ne pas être assez connu pour être utilisé dans tous les cas. Une périphrase est parfois utilisée à sa place, ce qui suggère que la notion a un sens mais que le terme n'est pas très répandu. Afin de respecter le principe d'économie de langage, les formulations condensées sont en général préférées aux formules longues. Cette idée a été théorisée par le linguiste 0 . Ducrot qui fait figurer la " loi d'économie » (1972 : 201) parmi les six « lois du discours » que nous devons respecter dans nos actes de communication. Il est donc d'usage d'utiliser un seul mot plutôt que plusieurs lorsque ce mot permet d'exprimer la même notion, sauf si l'on pense qu'il ne sera pas compris par les récepteurs du message. Il existe une périphrase qui aurait pu être remplacée par le terme "ecothriller» dans un extrait des critiques qui précèdent le roman Bones of Coral: «He has crafted a thriller with an eco-twist " (Hall 1991: 6). Cette formulation suggère que l'auteur ne connaît pas ce genre ou qu'il estime qu'il n'est pas familier au public qu'il vise. Le fait que le terme «ecothriller» apparaisse peu dans les paratextes de livres par ailleurs étiquetés comme tels par les critiques, suggère également que le terme n'est pas encore très répandu.

\subsection{Genèse du terme « ecothriller »}

8 L'origine de l'utilisation du terme "ecothriller" reste incertaine. Sa première occurrence n'a pas été identifiée précisément mais il semble possible d'indiquer qu'elle se situe dans les années 1980. La publication de Zodiac: The Ecothriller en 1988 paraît 
marquer une étape dans sa diffusion. Il n'y a pas pour autant lieu de croire que l'auteur de cette fiction soit le premier à l'avoir utilisé. Ce sous-titre montre cependant que c'est un terme que certains auteurs utilisent et trouvent pertinent pour la catégorisation de leurs œuvres. Aujourd'hui l'usage en est limité; il reste caractéristique de domaines assez restreints puisqu'il se trouve essentiellement sur les sites Internet et dans les journaux spécialisés, dans les commentaires de publications littéraires ainsi que dans les documents et sites Internet spécialisés dans la défense de l'environnement. Une recherche effectuée sur Google révèle par exemple que sur les trente premiers résultats, huit se trouvent sur des sites de vente en ligne, douze sur des sites de critiques littéraires, quatre sur des sites militants environnementaux, un sur un site de presse générale et cinq sur des sites divers. Par ailleurs, le terme commence également à se diffuser dans la presse plus générale ; c'est le sujet qui est maintenant abordé.

9 L'utilisation du terme par la presse générale n'est pas encore très répandue. Certains journaux ne l'emploient pas. Une recherche sur la version numérique de The Economist et The Daily Post reste par exemple infructueuse. En revanche, il apparaît sur la version numérisée du Times, du New York Times et de Newsweek. Dans la section «Arts and Entertainment» du Times se trouvent sept occurrences. La première occurrence répertoriée a été enregistrée en juin 2003. Le terme semble gagner en popularité à partir de 2006. Dans le New York Times, plusieurs occurrences du terme se rencontrent également dans les sections de critique littéraire et cinématographique. La première occurrence apparaît dès 1985. L'énonciateur le présente alors comme un terme dont il n'est pas sûr que la référence soit stabilisée. C'est ce que l'expression, «billed by its publisher as an ecothriller ", indique puisqu'il précise bien qu'il n'est pas lui même à l'origine de son utilisation. En 1995, une nouvelle occurrence du terme est attestée, avec cette fois encore une modalisation indiquant que la précision de celui-ci est remise en doute : «can best be described as an ecothriller ». Il reste ensuite seize occurrences du terme dont treize datent de 2004 ou plus tard et neuf de 2006 ou plus tard. Ces occurrences ne sont pas accompagnées d'une modalisation indiquant que la référence n'est pas stabilisée. Le terme semble désormais avoir acquis une légitimité.

Parmi ceux qui utilisent le terme, tous ne semblent pas en avoir la même définition. Sur le site d'Amazon par exemple, ne sont définis comme ecothrillers que des romans ayant pour thème le réchauffement climatique. S. Schollmeyer (2008) présente différentes problématiques environnementales pouvant relever du domaine des ecothrillers. Cette archiviste distingue les ecothrillers portant sur le thème du réchauffement climatique, tels que les romans de la trilogie de Kim Stanley Robinson $(2004,2005,2007)$ et Greenhouse Summer de Norman Spinrad, de ceux ayant pour thème la pollution, tels que Zodiac et Bones of Coral, de ceux enfin ayant pour thème la faune et la flore menacées, tels que les romans de la trilogie de Kirk Russel $(2004,2005,2007)$. C'est l'acception la plus large du terme dont nous défendons ici la définition. Pour la préciser, on peut se référer à l'encyclopédie en ligne Arts and Popular Culture :

an emerging sub-genre of the thriller in which the protagonist must avert or rectify an environmental or biological calamity - often in addition to dealing with the usual type of enemies or obstacles present in other thriller genres.

11 Pour expliquer cette position, une étude lexicographique du terme semble nécessaire. Le fragment de mot «eco » qui vient s'ajouter à un terme désignant un genre plus large, «thriller », peut être qualifié de fractomorphème. Il s'agit là d'un " fragment de lexie qui la représente dans un mot construit » (Tournier 2004 : 62). Dans ce cas, la lexie que «eco» représente dans le terme «ecothriller» est, bien entendu, «ecological». Or, le 
réchauffement climatique n'est pas un thème ayant une prééminence absolue dans le domaine de l'écologie même si c'est celui qui occupe le plus l'attention des médias et des politiques depuis environ six ans ${ }^{3}$. Ce n'est pas à ce thème particulier que le fractomorphème permet de référer. On peut plutôt penser qu'il porte «en quelque sorte l'essence sémantique du domaine » (Dury $2007: 64$ ), que l'on pourrait qualifier de gestion et protection des ressources naturelles. C'est donc tout ce qui a trait à ces questions qui peut être le thème principal des ecothrillers.

\subsection{Spécialisation ou non des auteurs?}

12 Une question qui revient régulièrement dans les interrogations au sujet de la FASP est celle de la spécialisation des auteurs. Dans de nombreux domaines de spécialité, il s'agit de déterminer si les auteurs sont des professionnels du domaine ou non (Petit 1999: $63 ; 2004: 67,175)$. Pourtant un grand nombre de métiers peuvent avoir une orientation environnementale. De nombreux termes désignant des métiers environnementaux peuvent ainsi être créés avec l'adjectif «environmental »: "environmental scientist », " environmental manager", "environmental lawyer ", "environmental economist», etc. De plus, il se pourrait que la professionnalisation ne soit pas la seule manière d'acquérir une spécialisation environnementale. Une personne qui milite pour une cause environnementale de manière très engagée mais non rémunérée doit-elle pour autant être considérée comme profane dans le domaine de spécialisation qu'est l'environnement? Et qu'en est-il d'une personne qui pratique régulièrement une activité physique de plein air et qui est amenée par ce biais à acquérir une connaissance fine de l'environnement? Ces quelques questions nous permettent de montrer que la problématique de la spécialisation dans le domaine environnemental est plus complexe qu'elle peut l'être dans les domaines le plus souvent cités comme représentatifs d'un genre de FASP.

13 Si l'on adopte un point de vue inclusif dans notre définition du spécialiste de l'environnement, on peut remarquer que plusieurs de nos auteurs peuvent être considérés comme des spécialistes du domaine. Nina Munteanu par exemple est présentée comme "an environmental scientist $»^{4}$. Kirk Russel reçoit l'étiquette de "an accredited professional in green building $»^{5}$ alors qu'Ian Irvine est directeur d'une entreprise qualifiée de "consulting firm, carrying out environmental studies $»^{6}$. Il s'agit donc de professions fort diverses mais qui ont toutes une dimension environnementale qui fait que ces auteurs peuvent être considérés en un certain sens comme des spécialistes du domaine. Pour ce qui est de Neal Stephenson, la situation est un peu différente puisqu'il n'a pas exercé en tant que professionnel de l'environnement, mais a obtenu un diplôme de géographie et de physique, ce qui lui permet probablement de parler de l'environnement de manière assez informée. Remarquons enfin que, comme dans toute catégorie de FASP, certains de nos auteurs d'ecothrillers n'ont pas de qualification particulière sur le sujet. Ainsi M. Crichton est un célèbre auteur de toutes sortes de fictions, N. Spinrad n'a étudié que l'écriture et a exercé en tant qu'auteur de romans et poésies ; enfin Phillip José Farmer est lui aussi uniquement écrivain.

14 Cette présentation des différentes caractéristiques de la FASP nous permet de vérifier que l'ecothriller respecte un grand nombre d'entre elles. En même temps, il ne s'agit pas d'un exemple prototypique puisque tous les critères permettant de définir la FASP ne 
sont pas respectés. Il se situerait plutôt dans ce qui est défini par Shaeda Isani comme les marges du genre :

as with all genres, differing degrees of "FASPness" exist, varying from the crystal clear at the core of the centripetal movement to the woolier and fuzzier forms

located on the fringes of the genre. (2004:34)

Ce sous-genre est encore très récent, ce qui pourrait expliquer le fait qu'il ne soit pas encore identifié par un large public. Nous postulons pourtant que les différents romans considérés ici ont suffisamment de caractéristiques communes pour qu'il soit possible de donner une définition plus précise du genre.

\section{Caractéristiques communes aux différents ecothrillers}

16 Le premier élément qui permet de caractériser le genre et qui se reflète dans son appellation, ecothriller, est l'aspect thématique. Le fractomorphème " eco» renvoie en effet au terme d'écologie, désignant à l'origine la science du rapport entre le vivant et son milieu mais venu à être utilisé avant tout dans un sens politique, que le Grand Robert définit comme suit :

Doctrine visant à une meilleure adaptation de l'homme à son environnement naturel, vivant (animaux, plantes) et non vivant, ainsi qu'à une protection de celuici ; courant politique défendant cette doctrine.

17 Le but des ecothrillers est donc de représenter l'homme luttant pour la protection de l'environnement. Dans chaque ecothriller une menace écologique est mise en scène, celle-ci pouvant être de nature très variée, comme il est montré dans la section 2.2. Ce critère thématique est le critère le plus évident, cité dans toutes les définitions du terme d'ecothriller. Il n'est pourtant pas le seul.

\subsection{Caractérisation des héros}

18 L'expertise environnementale peut s'acquérir chez des personnes pratiquant des métiers divers. Les héros de nos ecothrillers, qui ont souvent une forme d'expertise environnementale, ne pratiquent pas tous le même métier: avocat (State of Fear), inspecteur de police (Dark Rain),banquier (Ovum Factor), militant écologique (Freezing Point). Pourtant, sans qu'il y ait de règle générale à ce sujet, la majorité des héros d'ecothrillers exercent un type de profession spécifique. Mis à part les cas cités cidessus, tous les personnages principaux sont des chercheurs en sciences. C'est le cas dans Darkness Falls (Mills 2007), dans Whitewash (Kava 2007) et dans Desert Burial (Littlefair 2002). Pour ce qui est de The Swarm et de la trilogie sur le réchauffement climatique de K. S. Robinson, l'intrigue est située dans un laboratoire de recherche scientifique et les différents héros exercent donc tous cette profession.

Pour entrer un peu plus dans le détail de la caractérisation des héros, une présentation plus détaillée de ceux de la trilogie de K. S. Robinson peut s'avérer intéressante. Cette trilogie prend place à Washington où des scientifiques concernés par le réchauffement climatique s'efforcent d'y sensibiliser le monde politique et cherchent à développer de nouvelles technologies pour y faire face. Les personnages principaux sont des scientifiques travaillant pour la National Science Foundation ou comme conseillers scientifiques du gouvernement. Ils se heurtent à la frilosité des politiques et au manque 
de fonds publics comme privés. Tous se sentent personnellement engagés pour l'environnement. Certains de leurs traits d'identité ainsi que de ceux de personnes composant les réseaux sociaux dans lesquels ils évoluent en font des exemples typiques de la communauté des environnementalistes. Ainsi, la plupart des héros exercent un sport de plein air et de nombreux passages de cette trilogie sont consacrés à la description de ces différentes pratiques. Escalade, randonnée, kayak, surf, course à pied, frisbee... toutes ces activités sont représentées comme conduisant ceux qui les pratiquent à ressentir une sorte de fusion avec la nature, qui n'est pas sans lien avec leur engagement personnel en faveur de sa protection. Le passage suivant décrit une séance d'escalade de Frank, l'un des personnages principaux, et permet d'illustrer le sentiment de communion avec la nature qu'ils éprouvent :

Now as he tumbled down to the small tumble of big boulders at the foot of the cliff, chalked his hands, and began to climb the fine-grained old schist of the route, he cheered up. He focused on his immediate surroundings to a degree unimaginable when he was not climbing. It was like the math work, only then he wasn't anywhere at all. Here, he was right on these very particular rocks. (Robinson $2004: 86$ )

Dans le passage suivant, le même personnage pratique la course à pied : «Thus the joy of running in the forest, giving him little glimpses of the great unconscious mind» (Robinson 2005 : 143). Sa pratique sportive acquiert une dimension quasi spirituelle. L'auteur parvient à évoquer le lien traditionnel qui existe entre la communauté des sportifs de plein air et celle des environnementalistes ${ }^{7}$.

21 Une autre communauté présente dans la trilogie peut être considérée comme traditionnellement associée au mouvement des environnementalistes, celle des bouddhistes. Dès le premier livre, Anna, l'une des héroïnes principales, fait la connaissance d'un groupe de bouddhistes qui se réfugient à Washington à la suite de l'ensevelissement de leur île qui est une conséquence du réchauffement climatique. Trois des moines bouddhistes jouent un rôle dans l'intrigue; ils conduisent les scientifiques à progresser dans leur réflexion sur la protection de la nature en les amenant à repenser fondamentalement leur rapport avec elle. Frank sort d'une conférence intitulée "The purpose of science from the Buddhist perspective" avec une nouvelle certitude formulée en ces termes : " an excess of reason is itself a form of madness " (Robinson 2004: 268). Cet aspect du roman permet de reprendre un autre thème important dans la construction identitaire des environnementalistes. Il s'agit de l'intérêt qui les pousse vers des cultures non occidentales présentées comme ayant gardé une harmonie originelle avec la nature. Cet intérêt a également amené des peuples minoritaires à mettre en avant cette problématique dans leur lutte pour la défense de leurs droits afin d'atteindre un large public peu sensibilisé à leurs causes identitaires (Dawson $2000: 23$ ).

22 Cette caractérisation des héros de la trilogie de K. S. Robinson montre qu'ils présentent de nombreux traits identitaires typiques des environnementalistes et que ces romans offrent donc une représentation assez réaliste de cette communauté.

\subsection{Caractérisation des « ennemis » de l'environnement dans les ecothrillers}

Puisqu'une catastrophe écologique est toujours au centre de l'intrigue dans les ecothrillers, on peut penser que c'est elle qui joue le rôle de l'ennemi à combattre. Dans l'article du Library Journal, l'ecothriller est présenté en ces termes: "These novels have 
replaced the cold war tensions of the classic spy thriller with our struggle to survive ecological threats» (Schollmeyer 2008). Cette menace écologique se décline sous plusieurs formes. Il peut s'agir d'un virus incontrôlable comme dans Terminator Gene (Irvine 2003) et Darwin's Paradox (Munteanu 2007). Ce type d'intrigue semble inspiré d'événements du monde réel, ou du moins d'événements ayant occupé une place importante dans les médias, comme la grippe aviaire ou le phénomène plus récent de grippe H1N1. Il peut s'agir d'un phénomène moins réaliste, donnant un caractère de science-fiction au roman. Ainsi, dans The Swarm, l'ennemi à combattre est une force du mal qui s'incarne dans différents animaux, venus du fond des océans et hostiles aux humains.

Mais le conflit qui est au centre de ces thrillers n'oppose pas toujours les hommes à une force naturelle. Les tensions inter-humaines compliquent la situation et rendent la résolution des problèmes encore plus difficile. Dans le monde réel, un conflit opposant écologistes engagés et dirigeants, qu'ils soient politiciens ou hommes d'affaires, est souvent mis en évidence par la presse. Ce conflit traditionnel est le terreau dans lequel les auteurs d'ecothrillers semblent puiser. Les héros sont souvent des individus qui s'engagent pour une cause environnementale et qui se trouvent impuissants face à des personnages dirigeant le monde sans se soucier des problèmes environnementaux. Dans la grande majorité des cas, ceux qui détiennent le pouvoir politique et financier sont présentés sous un jour défavorable et ce sont eux que les héros sont amenés à combattre.

Des passages de la trilogie de K. S. Robinson dans lesquels ceux qui détiennent le pouvoir sont présentés d'un point de vue critique permettent de le montrer. Citons par exemple la remarque suivante concernant ceux qui détiennent l'argent qui pourrait aider à financer la recherche en faveur de l'environnement : " Their game was high-risk, high-return, long-range investment. This was not a kind of investment that banks would make, nor anyone else in the loaning world. The risks were too great, the returns too distant" (Robinson 2004: 216). Le portrait des politiques fait tout d'abord apparaitre leur réticence au changement ; on les voit par exemple faire semblant d'accepter le message des scientifiques concernant les efforts nécessaires pour faire face au réchauffement climatique, pour finalement céder aux intérêts du monde financier. Ce passage qui résume la politique de la Maison Blanche permet de le souligner.

The administration's first science advisor had been sent packing for saying that global warming might be real and not only that, amenable to human mitigations. That went too far for this administration. Their line was that no one knew for sure and it would be much too expensive to do anything about even if they were certain it was coming. (Robinson $2004: 155$ )

Un débat sur la manière dont on peut réformer le système politique pour faire face au réchauffement climatique mène à la réflexion suivante de l'un des personnages: «the economists should be trying to invent an honest accounting system that doesn't keep exteriorizing costs. When you exteriorize costs onto future generations you can make any damn thing profitable, but it isn't really true » (Robinson 2005 : 132). Le système économique en place et la rationalité sur laquelle il repose sont présentés dans ce roman comme des obstacles fondamentaux à la résolution des problèmes écologiques.

Ces exemples montrent donc ceux qui détiennent le pouvoir, cible traditionnelle des écologistes, sous un jour plutôt négatif. Pourtant, ce n'est pas toujours le point de vue des écologistes qui est adopté. Parfois, ce sont au contraire eux qui sont présentés comme la menace principale. Les catastrophes écologiques apparaissent alors comme 
des illusions dont ils sont en quelque sorte les instigateurs. Ainsi, dans Darkness Falls, les méchants sont des écologistes extrémistes et dans State of Fear, le réchauffement climatique est présenté comme une invention d'éco-terroristes qui provoquent euxmêmes les phénomènes semblant prouver son existence. Les ecothrillers ne nous donnent pas une vision totalement manichéenne de la crise puisque les points de vue adoptés à son sujet sont divers.

\subsection{Un substrat scientifique essentiel}

La définition de l'ecothriller donnée par S. Schollmeyer et citée plus haut peut donc sembler trop réductrice. Les héros ne sont pas toujours des individus qui se battent pour défendre l'environnement et qui parviennent à surmonter une crise écologique majeure. Dans certains cas, les forces du mal à combattre sont les écologistes et la crise qu'ils provoquent volontairement. La seule remarque d'ordre général qui s'applique à tous les romans est qu'ils offrent tous une vision de la crise écologique, des différents acteurs impliqués dans cette crise et des relations que ces acteurs entretiennent entre eux. Cet aspect des ecothrillers correspond à l'un des éléments de définition donnés par M. Petit pour la FASP : «qui ne montre pas seulement l'opposition classique entre les bons et les méchants mais décrit les mécanismes de ces forces de subversion d'un point de vue technique » (1999: 67). Bien que les points de vue soient différents, c'est bien toujours une description technique du problème qui est présente dans les ecothrillers. Les héros ne sont pas toujours des scientifiques mais l'importance de la science est apparente. Dans de nombreux cas, ce qui est mis en avant comme un point fort par les critiques qui commentent des ecothrillers c'est le caractère précis de la science présentée et l'authenticité des sources sur lesquelles elle se fonde. Sur la quatrième de couverture de Zodiac figure: "in creating this all too conceivable story of science and industry running amok, Stephenson puts his technological knowledge elegantly to use » et sur celle de The Ovum Factor (Zimmerman 2008), « Emotionally satisfying for the reader [...] plus the science is very interesting and credible».

Dans Forty Signs of Rain, le premier volume de la trilogie de K. S. Robinson, de nombreux passages décrivent des phénomènes physiques liés au réchauffement climatique: chaleur à New York, ouragans, montée des océans. Non seulement l'auteur utilise des termes précis et techniques pour désigner ces phénomènes, mais certains passages, en italique au début des chapitres, font figure de véritables explications scientifiques. Ces explications ne se trouvent donc pas dans le corps du roman mais leur importance est mise en avant par ce détachement. Il ne s'agit pas de décrire en détail les phénomènes climatiques évoqués. Le public cible de ce type de roman n'est pas forcément en mesure d'aborder une analyse très précise des phénomènes et les explications sont donc plutôt vulgarisées. Ces passages permettent néanmoins de souligner le caractère réaliste $d u$ roman qui s'appuie bien sur des études scientifiques pour construire son intrigue. Afin d'illustrer cette idée nous pouvons citer le début de Forty Signs of Rain :

The Earth is bathed in a flood of sunlight. A fierce inundation of photons - on average, 342 joules per second per square meter. 4185 joules (one Calorie) will raise the temperature of one kilogram of water by one degree Celsius. If all this energy were captured by the Earth's atmosphere, its temperature would rise by ten degrees Celsius in one day.

Luckily much of it radiates back to space. How much depends on albedo and the chemical composition of the atmosphere, both of which vary over time. (Robinson $2004: 3)$ 
30 spécialisé, cohabitent avec des expressions plus littéraires comme «bathed in a flood of sunlight ». Le texte est dans l'ensemble compréhensible pour un public non avisé.

31 un élément essentiel de la composition des ecothrillers. Une interrogation au sujet de l'authenticité des sources et plus généralement des rapports entre le réel fictionnel et le réel scientifique paraît, dans ce contexte, présenter de l'intérêt.

\section{Contiguïté entre le réel fictionnel et le réel catastrophique prédit par la science}

Le caractère hautement scientifique de chacun de ces romans pourrait signifier que leurs conclusions vont dans le même sens pour ce qui est de leur vision de la crise écologique. En effet, on a tendance à penser que ce que nous révèle la science est certain et n'est pas sujet à controverse. Pourtant tous les scientifiques n'aboutissent pas aux mêmes conclusions malgré la rigueur de leurs méthodes. Ces dissensions existent dans tous les domaines, mais sont particulièrement flagrantes dans le domaine de l'environnement. Depuis la publication du célèbre ouvrage de Rachel Carson, Silent spring, qui dénonçait le caractère biaisé des études visant à démontrer que les pesticides n'ont pas d'effets nocifs sur l'environnement, le caractère partial des positions de certains scientifiques a été démontré et des débats font rage entre scientifiques visant à démontrer l'ampleur de la menace écologique et ceux qui restent sceptiques au sujet de l'urgence de la situation. Le thème du réchauffement climatique est très représentatif de ces tiraillements. La mise en place de l'Intergovernmental Panel on Climate Change (IPCC), rassemblant scientifiques et gouvernements du monde entier pour étudier la validité des différentes théories sur le réchauffement climatique, répond à un besoin pressant : il s'agit de tenter de parvenir à un consensus malgré la diversité des positions. Le rapport publié par cet organisme en $2007^{8}$ a marqué les esprits en démontrant que le réchauffement climatique était bien réel et quasi certainement lié à l'activité de l'homme. De nombreuses incertitudes demeurent néanmoins quant à l'ampleur et à la vitesse de ce réchauffement. Elles amènent souvent les scientifiques à prôner des attitudes tout à fait divergentes au sujet des solutions politiques envisageables.

\subsection{Une littérature engagée?}

33 Les divergences scientifiques sur l'environnement replacent les solutions dans le champ politique et font des ecothrillers des outils médiatiques destinés à sensibiliser le grand public à la menace écologique. Dans la plupart des cas, il s'agit d'arriver à ce que les lecteurs prennent conscience de l'ampleur de la menace écologique et de l'horreur de ses conséquences possibles. De nombreuses critiques mettent en évidence le caractère effrayant des ecothrillers lié à leur très grand réalisme, comme si les crises écologiques qui y sont décrites s'apparentaient en tous points à celle que nous pourrions bien vivre d'ici quelques années : "frighteningly realistic " au sujet de Dark Rain, «Stephenson captures the New World so perfectly that one almost thinks that it is already there », sur la quatrième de couverture de Zodiac et « its apocalyptic scenarios and powerful ending seem inevitable in the real world" sur la quatrième de couverture de Greenhouse 
Summer. Parfois au contraire, comme dans State of Fear notamment, il s'agit de dénoncer la crise écologique comme une construction sociale et non comme un phénomène physique attesté. Dans les deux cas, la fiction peut être perçue comme ayant une fonction persuasive dans un débat écologique à dimension internationale. Les ecothrillers semblent donc avoir une dimension militante.

Par définition, un ouvrage de FASP vise à être lu par un large public. L'impact de ces ouvrages en est d'autant plus important. Dans son livre intitulé The Revenge of Gaïa, le scientifique britannique James Lovelock déplore l'influence de certains auteurs de fiction sur l'opinion publique. Faisant référence au roman sur le réchauffement climatique de M. Crichton, State of Fear, il montre qu'un roman de ce type peut avoir des conséquences néfastes.

I take Michael Crichton's opinions seriously, not because they are true, but because he is such a good storyteller [...]. The public is much more likely to be influenced by writers like Michael Crichton than they are by scientists. Fiction writers and film producers should ask themselves if they are sure that what they say is true before succumbing to the overriding imperative of the storyline; this is more important than ever before now that we face deadly change. (Lovelock $2006: 48$ )

J. Lovelock souligne le rôle essentiel que les ecothrillers pourraient avoir dans la prise de conscience du public et donc dans la résolution de la crise écologique. Il considère ici l'impact de ce type de roman sur un public de non-initié, que l'on pourrait décrire comme «naïve FASP reader » pour reprendre les termes de S. Isani (2009: 50). L'effet est très différent sur le « specialist FASP reader » (Ibid. : 51) dont J. Lovelock lui même est un exemple. Les réactions de spécialistes du domaine ont d'ailleurs été vives et critiques suite à la publication de State of Fear (Genty 2009 : 102).

\subsection{Une leçon sur l'avenir}

Cette idée de J. Lovelock pourrait nous conduire à une réflexion sur les rapports particuliers qu'entretiennent le réel et la fiction dans les romans de FASP. Le fait que ces romans participent à un débat d'ampleur internationale bien réel signifie qu'ils pourraient avoir une fonction éducative ou persuasive. Or pour persuader le public, il faut à la fois donner des explications convaincantes et parvenir à le toucher de manière émotionnelle. On retrouve ici les traditionnelles catégories argumentatives d'Aristote. Dans La Rhétorique, Livre I, cet auteur distingue dans les discours délibératifs les preuves logiques de celles qui s'appuient sur le pathos et l'ethos. Puisque «la passion c'est ce qui, en nous modifiant, produit des différences dans nos jugements » (Livre 2, chap. 1, 8), il importe, pour obtenir l'adhésion de son auditoire ou de son lectorat, de faire appel aux passions de celui-ci. De ce point de vue, on peut remarquer que de nombreux scientifiques qui ont écrit au sujet de la crise environnementale se sont efforcés de rendre leurs ouvrages compréhensibles pour un public large et d'utiliser des arguments qui s'appuient sur sa capacité à s'émouvoir. Une méthode qui est alors souvent adoptée est celle consistant à introduire des fables ou de petites histoires pour que le lecteur se représente de manière plus intense l'ampleur des conséquences possibles de la crise écologique. R. Carson, qui a écrit un livre considéré comme un jalon dans la prise de conscience environnementale d'un large public, présente une brève fable qui sert à illustrer la tristesse possible d'un monde sans oiseau. Son premier chapitre, intitulé "A Fable for Tomorrow», débute par la phrase : «There was once a town in the heart of America where all life seemed to live in harmony with its surroundings " (Carson 
1962 : 1). Les paragraphes suivants sont consacrés à la description de ce lieu idyllique mais, comme dans toute fable, les héros doivent affronter l'adversité :

Then a strange blight crept over the area and everything began to change. Some evil spell had settled on the community: mysterious maladies swept the flocks of chicken; the cattle and sheep sickened and died. Everywhere was a shadow of death. (Carson $1962: 2$ )

37 J. Lovelock présente également la terre comme un être vivant qui aurait contracté une maladie. Il la surnomme Gaïa, nom de la déesse de la terre dans la cosmogonie grecque, ce qui permet de souligner son caractère vivant. Il explique cette stratégie rhétorique en ces termes:

Metaphor is important because to deal with, understand, and even ameliorate the flux we are now in over global change requires us to know the true nature of the Earth and imagine it as the largest living thing in the solar system, not something inanimate. (Lovelock $2006: 17$ )

$38 \mathrm{Au}$ début de son ouvrage intitulé L'incandescent, Michel Serres (2005) introduit également une fable afin d'illustrer l'importance de certaines propriétés naturelles pour la communauté des humains.

39 Le fait que des scientifiques utilisent ce type de procédé rhétorique pourrait nous conduire à former une hypothèse, qui restera pour l'instant à l'état d'hypothèse étant donné le cadre limité de cette étude, sur les caractéristiques des communications sur la crise écologique visant un large public. Elles semblent composées d'une association d'éléments scientifiques et d'éléments visant à produire des émotions. Ces caractéristiques correspondent en tout cas à celles des communications faîtes dans le cadre de l'éducation environnementale. Selon la définition du Programme des Nations Unies pour l'Environnement, cette dernière est une éducation qui promeut des attitudes et des systèmes de valeurs produisant un comportement éthique vis-à-vis de l'environnement $^{10}$. Ce qui peut poser problème dans ce type de pratique éducative c'est qu'elle se propose de sensibiliser aux questions environnementales et de ce fait s'adresse aux fonctions émotives des individus ainsi qu'à leurs fonctions cognitives. Cette idée permettrait de développer une réflexion éventuelle sur les relations particulières entre la fiction et la réalité dans les ecothrillers, puisque ces derniers pourraient être vus comme entrant dans une catégorie similaire à celle des textes scientifiques à destination d'un large public sur l'environnement. Cette hypothèse nous permet d'envisager les effets des ecothrillers sur le monde réel, puisque loin d'être de simples fictions, ces romans viseraient à persuader le public d'adopter le point de vue de l'auteur sur la crise environnementale. Ils auraient donc un effet potentiel sur le public, et puisque la résolution de la crise environnementale dépend en grande partie de l'adoption d'une certaine attitude vis-à-vis de l'environnement par un large public, ces romans pourraient avoir un effet sur le monde réel. En tant que miroir, jamais totalement fidèle mais toujours représentatif du monde réel, l'ecothriller pourrait donc conduire à une prise de conscience et par ce biais, l'image réfléchie pourrait provoquer un changement dans l'image initiale.

L'étude de Forty Signs of Rain nous permet de donner corps à cette idée en nous appuyant sur un exemple précis. Les héros y sont tous des scientifiques ayant conscience $\mathrm{du}$ fait que la crise écologique peut être sérieuse. Pourtant ils sont confrontés à des politiciens qui se méfient du changement et sont hermétiques à toute déclaration catastrophiste. La fin du roman met pourtant en scène la catastrophe annoncée. En utilisant les réflexions de Jean-Pierre Dupuy, philosophe dont les travaux 
sont centrés autour de la définition de la catastrophe, nous voudrions montrer en quoi cet ecothriller pourrait être vu comme ayant un rôle à jouer dans le monde réel, grâce à son effet de miroir.

Une étude de textes écrits juste après qu'une catastrophe a eu lieu conduit J.-P. Dupuy à la décrire comme "le surgissement de l'impossible dans le réel» (2002:12). Mais ce n'est pas seulement le présent qui change radicalement lors de ce surgissement de la catastrophe dans le réel. Le passé lui-même se transforme puisqu'il paraît soudain contenir la possibilité de la catastrophe imminente, sans quoi celle-ci n'aurait pu se produire. J.-P. Dupuy défend l'idée que nous devons introduire la possibilité de la catastrophe dans notre présent pour mieux pouvoir la prévenir. Le roman de K. S. Robinson apparaît comme une confirmation de ce que prône J.-P. Dupuy. En effet, les personnages décrits ici sont volontairement des individus qui nous ressemblent. Ils vivent dans un monde semblable en tous points à notre monde contemporain et non à un futur lointain, menaçant mais aisément négligeable. Ils perçoivent la catastrophe comme le surgissement de l'impossible dans le réel: " he had crossed over into a space where the real world had taken on all the qualities of a dream " (Robinson 2004:357). Ce passage dans de l'irréel, du rêve, de l'impossible dans le réel est décrit ici de manière réaliste. L'auteur semble viser l'identification du public avec ses personnages soumis à la catastrophe, et il pourrait contribuer à prévenir la catastrophe, s'il était pris au sérieux, si cet ecothriller était considéré comme un reflet du réel.

\section{Conclusion}

Au terme de cette étude, il peut être intéressant de tenter de déterminer si nous avons bien affaire ici à un nouveau genre textuel. Je m'appuie ici sur l'étude qu'A. Saber consacre à la définition du genre. Il considère que quatre critères aident à établir ce qu'est un genre et il évoque l'existence de différents degrés de cristallisation générique :

[L]e genre est un dispositif énonciatif inscrit dans une situation sociale et culturelle; il est produit et géré par des communautés discursives, qui peuvent revêtir une forme professionnelle ou disciplinaire ; il est marqué par des régularités langagières diverses; en outre, un " macro-acte de langage " global lui est associé. À partir de ce "cahier des charges " générique, il semble possible de décrire la façon dont un texte se cristallise en un genre donné, en établissant un " prototype » de celui-ci (la satisfaction, même partielle, des critères que nous venons de définir, détermine cette cristallisation générique - qui peut demeurer incomplète ou simplement « tendancielle»). (Saber $2006: 48$ ) précisée, bien que de manière assez vague, dans le sens où son développement semble intimement lié à notre époque contemporaine où l'on observe une croissance de préoccupations écologiques chez le grand public. Les ecothrillers semblent «marqué(s) par des régularités langagières " puisqu'on y trouve une concentration importante de vocabulaire scientifique au sujet de la crise écologique. La communauté discursive dans laquelle on produirait des exemplaires prototypiques de ces romans serait celle des environnementalistes; de nombreux auteurs de tels romans sont en effet engagés en ce sens. Identifier un "macro-acte de langage " à l'œuvre dans les ecothrillers semble également possible. En effet, il s'agit dans tous les cas de mettre en scène la crise 
écologique et de se prononcer à son sujet, souvent à la fois d'un point de vue scientifique et politique.

Il est donc possible de tenter de préciser ce qu'est le genre de l'ecothriller grâce à ces quatre critères mais la définition prototypique en reste encore assez imprécise. Toute tentative de caractérisation plus aboutie semble buter sur certaines exceptions tant les scénarios et les points de vue présentés par les auteurs diffèrent d'un roman à l'autre. Peut-être s'agit-il d'un genre en cours de cristallisation générique. Pourtant l'intérêt de l'usage pédagogique de ce type de roman pour des étudiants spécialistes de disciplines environnementales, ou pour des étudiants d'anglais de spécialité tentant de définir le domaine environnement, ne fait pas de doute. Mis à part le fait qu'il permet d'introduire de nombreux termes, il donne des renseignements sur les communautés qui agissent dans le contexte de la crise écologique, sur leur caractérisation identitaire, ainsi que sur divers aspects de la crise elle-même. Il nous invite également à entamer une réflexion au sujet de cette dernière, réflexion qui dans certains cas semble devoir déboucher sur une prise de conscience de son importance comme dans le cas de la trilogie de K. S. Robinson.

L'étude des ecothrillers nous paraît finalement intéressante du point de vue de la recherche en FASP. Si les premières catégories d'ouvrages étudiés dans le cadre de la FASP étaient caractéristiques des années 1990, les ecothrillers seraient plutôt une FASP des années 2000. Leur étude pourrait refléter une évolution dans les domaines spécialisés choisis comme objets d'analyse dans le cadre de l'anglais de spécialité. Si M. Petit observe que ce qui permet une «représentation du professionnel [...] à la fois plus large et plus approfondie» ( $2004: 17)$ dans les premiers ouvrages identifiés comme FASP est un « acquis culturel » (Ibid.) déjà présent chez le grand public, on peut penser que les ecothrillers n'en sont qu'aux premiers stades de leur développement et qu'il est encore trop tôt pour que le public soit familiarisé à ce point avec les problématiques écologiques et les milieux professionnels qui se développent autour de ces questions. Le degré de spécialisation de ce type de fiction pourrait s'approfondir par la suite. Peut-être serait-il alors possible de voir si les ecothrillers évoluent vers un prototype générique plus aisément identifiable.

La présente étude avait pour objectif d'introduire ce type d'objet plutôt que d'offrir des conclusions abouties à son sujet. Il reste néanmoins qu'il nous a paru intéressant d'observer les effets de miroir à l'œuvre dans ce type de fiction. Comme évoqué, la réalité aurait un effet réfléchissant sur la FASP en ce qu'une évolution des domaines spécialisés considérés comme importants influencerait le type de production dans ce domaine. Mais il se peut que l'effet de miroir n'aille pas uniquement dans ce sens. Peuton aller jusqu'à dire que la fiction tente d'influencer le réel? En représentant les catastrophes écologiques dans un contexte très réaliste comme "le surgissement de l'impossible dans le réel », certains ouvrages de FASP pourraient contribuer à accélérer la prise de conscience du public au sujet des problématiques environnementales et par ce biais contribuer à une amélioration de la situation de l'environnement. En ce sens, ils pourraient servir d'outils pédagogiques forts utiles pour des formateurs en éducation environnementale. 


\section{BIBLIOGRAPHIE}

\section{Sources primaires}

Corderoy, Conor. 2006. Dark Rain. Londres : Macmillan New Writing.

Crichton, Michael. 2005. State of Fear. Londres : HarperCollins.

Dionne, Karen. 2008. Freezing Point. New York : Penguin Group.

Hall, James W. 1991. Bones of Coral. New York : Knopf Doubleday.

Irvine, Ian. 2000. The Last Albatros. East Roseville, Australie : Simon \& Schuster.

Irvine, Ian. 2003. Terminator Gene. East Roseville, Australie : Simon \& Schuster.

Irvine, Ian. 2004. The Life Lottery. East Roseville, Australie : Simon \& Schuster.

Kava, Alex. 2007. Whitewash. Hortonville, WI : Mira Publishing.

Littlefair, Brian. 2002. Desert Burial. New York : Henry Holt and Company.

Mills, Kyle. 2007. Darkness falls. New York : Vanguard Press.

Munteanu, Nina. 2007. Darwin's Paradox. Calgary, Alberta, Canada : Dragon Moon Press.

Robinson, Kim Stanley. 2004. Forty Signs of Rain. New York : Bantam Dell.

Robinson, Kim Stanley. 2005. Fifty Degrees Below. New York : Bantam Dell.

Robinson, Kim Stanley. 2007. Sixty Days and Counting. New York : Bantam Dell.

Russel,Kirk. 2004. Shell Games. San Francisco : Chronicle Books LCC.

Russel, Kirk. 2005. Night Game. San Francisco : Chronicle Books LCC.

Russel, Kirk. 2007. Dead Game. San Francisco : Chronicle Books LCC.

Schatzing, Frank. 2006 [2004]. The Swarm. New York: William Morrow.

Spinrad, Norman. 2000. Greenhouse Summer. New York : Tor Books.

Stephenson, Neal. 1988. Zodiac: The Eco-thriller. New York : Atlantic Monthly Press.

Zimmerman, Marvin. 2008. The Ovum Factor. Austin, TX : Synergy Books.

\section{Références}

Aristote. 1922. Poétique et Rhétorique, traduction d'Émile Ruelle, version numérisée consultée le 27/04/09 <http://remacle.org/bloodwolf/philosophes/Aristote/ rheto1.htm>.

Carson, Rachel. 1962. Silent Spring. Boston, MA : Houghton Mifflin.

Charaudeau, Patrick et Dominique Maingueneau (dir.). 2002. Dictionnaire d'analyse de discours.

Paris : Seuil.

Dawson, Jane. 2000. « The two Faces of environmental justice: Lessons from the eco-nationalist phenomenon ». Environmental Politics 9/2, 22-60.

Ducrot, Oswald. 1972. Dire et ne pas dire. Principes de sémantique linguistique. Paris : Hermann.

Dupuy, Jean-Pierre. 2002. Pour un catastrophisme éclairé. Paris : Seuil.

Dury, Pascaline. 2007. « La déterminologisation du formant eco- et la terminologisation de

l'adjectif carbon neutral un aperçu diachronique de la migration des termes ». Actes de la Conférence 
TIA'07 - $7^{e}$ Conférence « Terminologie et Intelligence Artificielle », 2007 Grenoble : Presses

Universitaires de Grenoble, 61-70.

Genty, Stéphanie. 2009. « Apparent truth and false reality: Michael Crichton and the distancing of scientific discourse ». ASp 55, 95-105.

Guggenheim, Davis. 2006. An Inconvenient Truth, Vidéo. Hollywood, CA : Paramount Classics and Participant Productions.

Isani, Shaeda. 2004. " The FASP and the genres within the genre ». In Petit, M. (dir.). Aspects de la fiction à substrat professionnel. Collection EA2025. Bordeaux : Université Bordeaux 2, 25-36

Isani, Shaeda. 2009. « Specialised fictional narrative and lay readership: Bridging the accessibility gap ». ASp 56, 45-65.

Lovelock, James. 2006. The Revenge of Gaïa. New York : Perseus Groups.

Petit, Michel. 1999. « La fiction à substrat professionnel : une autre voie d'accès à l'anglais de spécialité ». ASp 23-26, 57-81.

Petit, Michel (dir.). 2004. Aspects de la fiction à substrat professionnel. Collection EA2025. Bordeaux : Université Bordeaux 2.

Saber, Anthony. 2006 « Les milieux militaires américains et leurs discours ». Thèse en études anglaises, Université Bordeaux 2.

Schollmeyer, Shawn. 2008. « Apocalypse now? Summer ecothrillers ». Library Journal Online, consulté le 21/11/08 <www.libraryjournal.com>.

Serres, Michel. 2005. L'incandescent. Paris : Librairie Générale Française.

Stern, Nicholas H. 2007. The Economics of Climate Change: The Stern Review. Cambridge : Cambridge University Press.

Tournier, Jean. 2004 [1988]. Précis de lexicologie anglaise. Paris : Ellipses.

\section{Sources Internet}

<http://www.amazon.co.uk>. Consulté le 18/11/08.

<http://www.artandpopularculture.com>. Consulté le 27/04/09.

<http://www.dailypost.co.uk>. Consulté le 27/04/09.

<http://www.economist.com>. Consulté le 27/04/09.

<http://www.hachettebookgroup.com>. Consulté le 18/11/08.

<http://www.kirkrussellbooks.com>. Consulté le 18/11/08.

<http://www.newsweek.com>. Consulté le 27/04/09.

<http://www.paperbackswap.com>. Consulté le 21/11/08.

<http://www.timesonline.co.uk>. Consulté le 27/04/09.

<http://www.unep.org/training>. Consulté le 27/04/09.

<http://www2.loot.co.za>. Consulté le 21/11/08. 


\section{NOTES}

1. Voir les nombreux articles publiés dans la presse à sa mort en novembre 2008, qui le présentent tous comme le premier et le principal auteur de technothrillers.

2. D'après la définition du Grand Robert : «Ensemble des éléments éditoriaux qui accompagnent un texte publié et qui orientent la lecture (titre, dédicace, préface, notes, etc.) ».

3. Dans les discours politiques de Tony Blair pendant qu'il était au pouvoir par exemple, ce n'est qu'à partir de 2004 que le thème du réchauffement climatique devient prééminent par rapport aux autres questions écologiques. Avant cette date, il était cité à côté d'autres thèmes écologiques considérés comme tout aussi importants.

4. <http://www.amazon.com/gp/product/product-description/189694468X/ref=dp_proddesc_0? ie=UTF 8 \&n=283155\&s=books $>$ consulté le $21 / 11 / 08$.

5. <http://www.kirkrussellbooks.com/kirk.php> consulté le 21/11/08.

6. <http://www.hachettebookgroup.com/authors_Ian-Irvine-(1015232). htm> consulté le $21 / 11 / 08$.

7. De nombreuses associations environnementales ont été créées par des sportifs soucieux de protéger l'environnement naturel dans lequel ils évoluent. Celle qui a le plus de notoriété est la Surfrider Foundation, une ONG internationale de protection de la nature dont la priorité est de veiller à la propreté des océans. Pourraient être aussi cités l'Adirondack Mountain Club et la Montana Wilderness Association créés au début du siècle par des randonneurs.

8. Pachauri, R. K. et A. Reisinger (dir.), Contribution of Working Groups I, II and III to the Fourth Assessment Report of the Intergovernmental Panel on Climate Change. IPCC : Genève.

9. <http://www.amazon.co.uk/Dark-Rain-Conor-Corderoy/dp/023000010X> consulté le $21 / 11 / 08$.

10. <www.unep.org/training/>.

\section{RÉSUMÉS}

Une profusion de discours sur la crise écologique actuelle semble se manifester à tous les niveaux de la société (dans l'univers des médias, des entreprises, de l'éducation, dans la sphère politique...) ; il peut donc paraitre naturel qu'elle se manifeste également dans le domaine de la fiction. C'est ce dont témoigne le développement récent d'un nouveau genre de fiction spécialisée, l'ecothriller, dont l'auteur souhaite présenter les premiers éléments d'une définition. Tout d'abord l'origine de cette nouvelle étiquette générique est étudiée, puis elle est définie à travers l'analyse des points communs entre les différentes fictions qualifiées comme telles. Cette étude amène aussi à s'interroger sur le lien qui existe entre fiction et réel dans ce type de fiction à substrat professionnel. Ce lien peut être comparé à celui d'un double effet de miroir tant la production d'ecothrillers semble découler de faits réels et tant les auteurs de ces romans semblent viser à jouer un rôle politique ou éducatif dans le monde réel.

A high number of discourses on the current ecological crisis seem to be appearing in different areas of society - the media, the business world, the education sector, the political world... - and therefore quite naturally in the domain of fiction as well, as evidenced by the recent emergence of a new specialised fiction genre, the ecothriller, which the author proposes to map out here. She first identifies the origin of this new generic tag and the generic features that the different 
novels called ecothrillers share. The study then sets out to analyse the link between fiction and reality in this type of specialised genre and the double mirror effect obtained from the fact that ecothrillers seem to flow from real events and their authors seem to aim at playing a political or educational role in the real world.

INDEX

Mots-clés : crise écologique, ecothriller, environnementaliste, fiction à substrat professionnel Keywords : ecological crisis, ecothriller, environmentalist, specialised narrative

\section{AUTEUR}

\section{CAMILLE BIROS}

Camille Biros, agrégée d'anglais, est monitrice au département d'anglais de l'École Normale Supérieure de Cachan où elle donne des cours d'anglais à des spécialistes d'autres disciplines. Elle prépare une thèse de doctorat à l'Université Victor Segalen Bordeaux 2 sous la direction de Michel Petit et elle est membre de l'EA 4140. Ses recherches portent sur les discours spécialisés du domaine de l'environnement. Sa thèse traite plus spécifiquement des discours produits par les organismes liés à l'environnement au Royaume-Uni. camille.biros@laposte.net 